\title{
Development and clinical evaluation of a 3-step modified manipulation protocol for MRI-guided high-intensity focused ultrasound of uterine fibroids
}

\author{
Inez M. Verpalen ${ }^{1} \cdot$ Miranda van 't Veer-ten Kate ${ }^{1} \cdot$ Erwin de Boer $^{1} \cdot$ Rolf D. van den Hoed ${ }^{1} \cdot$ Joke M. Schutte $^{2}$. \\ Jeroen R. Dijkstra ${ }^{2}$ - Arie Franx ${ }^{3}$ - Lambertus W. Bartels ${ }^{4} \cdot$ Chrit T. W. Moonen $^{4}$ - Martijn F. Boomsma ${ }^{1}$
}

Received: 8 November 2019 /Revised: 12 February 2020 / Accepted: 25 February 2020

(C) European Society of Radiology 2020

\begin{abstract}
Objectives The clinical applicability of magnetic resonance image-guided high-intensity focused ultrasound (MR-HIFU) treatment of uterine fibroids is often limited due to inaccessible fibroids or bowel interference. The aim of this study was to implement a newly developed 3-step modified manipulation protocol and to evaluate its influence on the number of eligible women and treatment failure rate.

Methods From June 2016 to June 2018, 165 women underwent a screening MRI examination, 67 women of whom were consecutively treated with MR-HIFU at our institution. Group $1(n=20)$ was treated with the BRB manipulation protocol which consisted of sequential applications of urinary bladder filling, rectal filling, and urinary bladder emptying. Group 2 ( $n=47)$ was treated using the 3-step modified manipulation protocol which included (1) the BRB maneuver with adjusted rectal filling by adding psyllium fibers to the solution; (2) Trendelenburg position combined with bowel massage; (3) the manual uterine manipulation (MUM) method for uterine repositioning. A comparison was made between the two manipulation protocols to evaluate differences in safety, the eligibility percentage, and treatment failure rate due to unsuccessful manipulation.

Results After implementing the 3-step modified manipulation protocol, our ineligibility rate due to bowel interference or inaccessible fibroids decreased from $18 \%(16 / 88)$ to $0 \%(0 / 77)$. Our treatment failure rate due to unsuccessful manipulation decreased from $20 \%(4 / 20)$ to $2 \%$ (1/47). There were no thermal complications to the bowel or uterus.

Conclusions Implementation of the 3-step modified manipulation protocol during MR-HIFU therapy of uterine fibroids improved the eligibility percentage and reduced the treatment failure rate.

Trial registration Registry number NL56182.075.16

Key Points

- A newly developed 3-step modified manipulation protocol was successfully implemented without the occurrence of thermal complication to the bowel or uterus.

- The 3-step modified manipulation protocol increased our eligibility percentage for MR-HIFU treatment of uterine fibroids.

- The 3-step modified manipulation protocol reduced our treatment failure rate for MR-HIFU treatment of uterine fibroids.
\end{abstract}

Keywords Uterine fibroids $\cdot$ MR-guided interventional procedures $\cdot$ High-intensity focused ultrasound ablation $\cdot$ Patient selection

Electronic supplementary material The online version of this article (https://doi.org/10.1007/s00330-020-06780-2) contains supplementary material, which is available to authorized users.

Inez M. Verpalen

i.m.verpalen@isala.nl

1 Department of Radiology, Isala Hospital, Dokter van Heesweg 2, 8025 AB Zwolle, The Netherlands

2 Department of Gynecology, Isala, Zwolle, The Netherlands
3 Department of Obstetrics and Gynecology, Erasmus Medical Center, Rotterdam, The Netherlands

4 Image Sciences Institute, Imaging Division, University Medical Center Utrecht, Utrecht, The Netherlands 


$\begin{array}{ll}\text { Abbreviations } \\ \text { BRB } & \text { Bladder filling, rectal filling, bladder emptying } \\ \text { CE } & \begin{array}{l}\text { Contrast-enhanced } \\ \text { Magnetic resonance image-guided high- } \\ \text { MR-HIFU } \\ \text { intensity focused ultrasound }\end{array} \\ \text { MRI } & \begin{array}{l}\text { Magnetic resonance imaging } \\ \text { MUM }\end{array} \\ \text { Manual uterine manipulation } \\ \text { NPV } & \text { Non-perfused volume } \\ \text { SI } & \text { Signal intensity }\end{array}$

\section{Introduction}

Magnetic resonance image-guided high-intensity focused ultrasound (MR-HIFU) has been a treatment option for uterine fibroids since 2004. MR-HIFU has been proven to be safe and effective in reducing fibroid-related symptoms and fibroid size $[1,2]$. Although the treatment has emerged as the only non-invasive interventional therapy, MR-HIFU has not been widely adopted into regular care up to now. It seems plausible that a combination of factors is responsible for this such as technical limitations and clinical availability, and also familiarity of the gynecologist with such expertise. Technical limitations of MR-HIFU treatment include the duration of the treatment, the focal depth and maximum power of the HIFU system, and the relatively low eligibility percentage compared with hysterectomy and embolization [3, 4]. The number of referred patients with fibroids suitable for MR-HIFU therapy in previously reported studies ranged from 14 to $74 \%$ depending on the used selection guidelines [5-7]. A screening MRI examination is performed to assess technical eligibility, but it remains difficult to predict MR-HIFU treatment outcome based on imaging characteristics. Moreover, even when eligible patients undergo MR-HIFU therapy, the risk for an unsuccessful treatment persists. A frequent reason for ineligibility and failure of MR-HIFU therapy was the interposition of bowel loops in the acoustic pathway $[8,9]$. Interestingly, it was reported that interposed bowel loops occurred in the expected sonication path on the treatment day while there was no interposition present on the screening MRI examination [10]. This finding suggested a reversible situation; therefore, manipulation techniques have been widely implemented. The most common technique to displace bowel loops was described by Park et al This technique includes sequential applications of urinary bladder filling, rectal filling, and bladder emptying, which is also referred to as the BRB maneuver [10]. However, the problem of bowel interference can still persist when the BRB maneuver fails. In our center, a newly developed manipulation protocol was implemented which consisted of three different manipulation techniques. This gave us the opportunity to evaluate the difference between two manipulation protocols with respect to the eligibility percentage and treatment failure rate due to unsuccessful manipulation.

\section{Material and methods}

This prospective single-center study was conducted from June 2016 to June 2018. This study (registry number NL56182.075.16) was approved by our local Research Ethics Committee (number 16.0479). Information about the medical history of patients was retrieved from medical records. Informed consent was obtained from all patients.

\section{Patients}

All women presenting with fibroid-related symptoms at the gynecology outpatient clinic were evaluated for inclusion. Eligible patients were offered to undergo an MRI examination and their symptom severity and quality of life were assessed with a validated disease-specific questionnaire [11]. Inclusion criteria were uterine fibroids confirmed on ultrasound, age over 18 years, and pre- or peri-menopausal state. Exclusion criteria were as follows: pregnancy, calcification of the uterine fibroid, severe obesity, or MRI contraindications including contrast allergy. These inclusion and exclusion criteria remained the same, independent of the used manipulation protocol.

\section{Screening MRI examination}

The screening MRI protocol to assess patient suitability for MR-HIFU treatment consisted of three-dimensional (3D) T2weighted imaging and contrast-enhanced (CE) T1-weighted imaging immediately after administration of a contrast agent (DOTAREM $®, 0.2 \mathrm{~mL} / \mathrm{kg}$; gadoterate meglumine, $0.1 \mathrm{mmol} /$ $\mathrm{kg})$. All MRI examinations were evaluated by radiologists with MR-HIFU experience. The position of the uterus, the number of fibroids, fibroid location, the presence of bowel interference, and abdominal scar tissue were reported. One researcher (I.V.) performed all measurements on the screening MRI examination: the thickness of the subcutaneous abdominal fat layer, maximum diameter, and fibroid volume by semi-automatic segmentation in IntelliSpace Portal (ISP) software (Philips Healthcare). Signal intensity (SI) of the fibroid on T2-weighted (T2w) images was compared to the SI of the rectus abdominis muscle and the myometrium, and the fibroid was classified according to the Funaki classification [12].

\section{Patient selection for MR-HIFU}

Potentially eligible patients for MR-HIFU therapy were selected based on the screening MRI. Exclusion criteria for MRHIFU included current pregnancy, fat layer $<4 \mathrm{~cm}$, concomitant adenomyosis, suspicion of malignancy on screening MRI, no contrast-enhancement of the targeted fibroid on the T1weighted (T1w) image, maximum diameter of the dominant fibroid $<1 \mathrm{~cm},>10$ uterine fibroids (without one or two large 
dominant fibroids explaining the clinical symptoms of the patient), excessively high T2 signal of the targeted fibroid compared with the myometrium on the T2w image, or an inaccessible targeted uterine fibroid (despite the use of manipulation techniques, the depth of the posterior wall of the targeted uterine fibroid will be $>10 \mathrm{~cm}$ from the abdominal wall).

The choice to undergo MR-HIFU therapy was based on patient preference. In total, 165 women consecutively underwent a screening MRI examination during the study period, of which 99 patients seemed eligible and 67 women were consecutively treated with MR-HIFU (Fig. 1). To evaluate the influence of the used manipulation protocol on the eligibility percentage and treatment failure rate, two study cohorts were compared (Fig. 1). In group A, 88 women were screened, of which 41 seemed eligible and 20 patients consecutively underwent MR-HIFU therapy (group 1). If indicated, these first 20 patients received manipulation techniques according to protocol 1. In group B, 77 women were screened, of which 58 seemed eligible and 47 patients consecutively underwent MR-HIFU therapy (group 2). If indicated, these last 47 patients received manipulation techniques according to protocol 2.

\section{Patient preparation}

Pre-operative evaluation was performed before the MR-HIFU procedure for anesthetic risk assessment. All patients fasted $6 \mathrm{~h}$ prior to therapy and were asked to shave their lower abdomen and pubic region. Patient preparation was performed at the nursing department. Bowel preparation was done using a fast-acting Microlax micro-enema. A single-dose (1 tube of $5 \mathrm{~mL}$ ) was administered rectally. Patients received oral premedication which included paracetamol $1000 \mathrm{mg}$, diclofenac $100 \mathrm{mg}$, and oxycodone $10 \mathrm{mg}$. A Foley catheter and intravenous line were inserted directly before treatment.

Fig. 1 Flow diagram

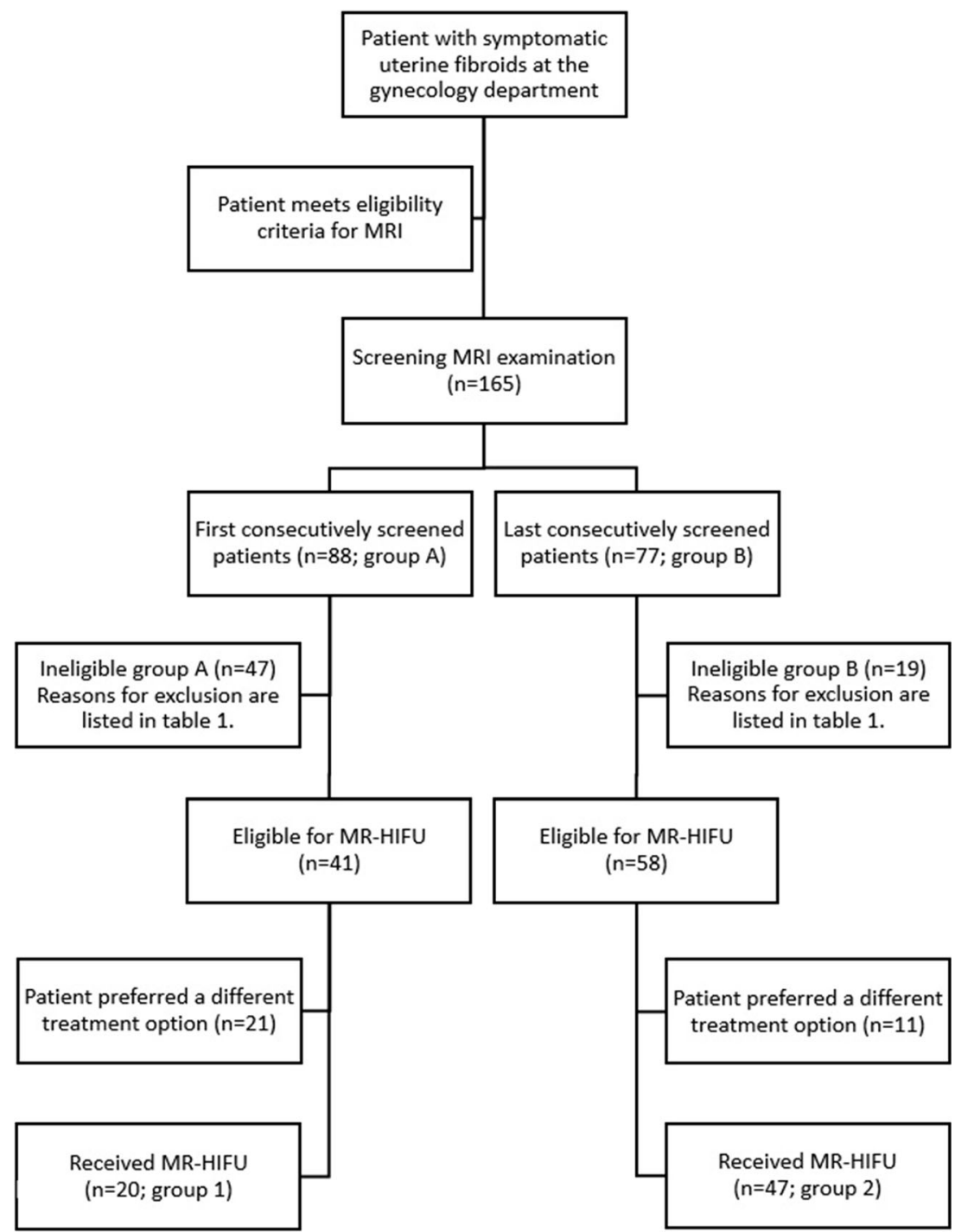




\section{Manipulation techniques}

When required, manipulation techniques were applied to achieve the optimal position of the targeted uterine fibroid to allow for complete ablation. The optimal position was defined as follows: targeted uterine fibroid being entirely accessible by the system without interposition of critical structures in the near and far fields of the beam pathway. At the start of the MR-HIFU treatment, patients were placed into prone position on the MRI table. A gel pad and degassed water were used for acoustic coupling. A survey MR image and T2w 3D planning images were obtained to reassess the location of the uterine fibroid. None of the manipulation techniques was applied if the anatomical location of the fibroid was optimal on the $\mathrm{T} 2 \mathrm{w}$ 3D planning images. Manipulation techniques were indicated when the targeted uterine fibroid was (partially) inaccessible or interposition of critical structures was observed in the beam pathway. These manipulation techniques were applied using two different protocols:

Protocol 1 ( $n=20$ treatments): the BRB maneuver which included sequential applications of urinary bladder filling, rectal filling, and urinary bladder emptying [10]. Bladder filling was performed by manually squeezing a plastic bag of saline connected to the side arm of a clamped Foley catheter. The amount of urinary filling ranged from 100 to $500 \mathrm{~mL}$ and was adjusted to patient tolerance. The rectum was filled with multiple syringes $(60 \mathrm{~mL})$ using a solution of ultrasound gel $(30 \mathrm{~mL})$ and saline solution $(30 \mathrm{~mL})$ via a rectal catheter. The amount of rectal filling ranged from 240 to $480 \mathrm{~mL}$, based on patient tolerance. To facilitate bladder emptying, natural drainage was allowed by declamping the catheter. The $\mathrm{BRB}$ maneuver required an additional $30 \mathrm{~min}$.

Protocol 2 ( $n=47$ treatments): the 3-step modified manipulation protocol.

1. The BRB maneuver with modified rectal filling in order to ensure a consistent and greater anterior displacement; compared with the above described BRB technique, the rectum was not filled with ultrasound coupling gel alone, but with a modified solution by adding 1 sachet $(3.4 \mathrm{~g})$ of psyllium fibers (Metamucil $\AA$ ) to the $60-\mathrm{mL}$ syringe. This BRB manipulation moved the fibroid anteriorly or displaced bowel loops (Fig. 2).

2. Trendelenburg position with bowel massage; patients were positioned into the Trendelenburg position to move the small bowel out of the pelvis (Fig. 3). Additionally, abdominal massage was performed on both sides of the lower abdomen with movements toward the upper abdomen. This technique was used when the uterine fibroid was located ventrally (anteverted uterus) and the BRB maneuver failed to succeed. The extra time spent on patient positioning was approximately $45 \mathrm{~min}$.
3. Manual uterine manipulation (MUM): a retroverted position of the uterus required manual repositioning into anteflexion. To prepare for MUM, the urinary bladder was filled with saline to prevent the interposition of bowel loops during anteversion. Afterwards, we fixated the position of the uterus (Fig. 4) with rectal filling and/or the use of a disposable plastic vaginal speculum (Bridea Medical). The speculum was kept out of the beam pathway to avoid potential interference in the ultrasonic energy field. When the uterine fibroid was located dorsally (anteverted uterus) in the recto-sigmoid region (or when the BRB maneuver failed to succeed), the operator used the MUM method for repositioning of the uterine fibroid itself (Fig. 4). On average, MUM required $15 \mathrm{~min}$.

\section{MR-HIFU treatment}

MR-HIFU treatments were performed using a clinical HIFU system (Sonalleve, V1, Profound Medical Inc.) integrated into a 1.5-T MR scanner (Achieva; Philips Healthcare). Three radiologists (R.v.d.H.; E.d.B.; M.v.t.V.t.K.) carried out all the MR-HIFU procedures. Procedures were performed on an outpatient basis under conscious sedation using propofol-fentanyl. During MUM, a bolus of propofol was given to induce moderate-deep sedation followed by supplemental boluses if required. If necessary, Buscopan ${ }^{\circledR}$ (butibrometo escopolamina) was administered intravenously (multiple times) to decrease bowel motion because the HIFU system is sensitive to motion artifacts. Respiration-related motion artifacts were managed with breath hold instructions, especially when the targeted fibroid was small $(<3 \mathrm{~cm})$. Post-treatment CE-T1w images were obtained to visualize the treatment result, called the non-perfused volume. The non-perfused volume (NPV) post-treatment was measured and the NPV\% was calculated (the NPV divided by the fibroid volume).

\section{Results}

Group $1(n=88)$ resulted in an ineligibility percentage of $53 \%$ (47/88) based on the screening MRI (Table 1), of which $18 \%$ $(16 / 88)$ was due to the fibroids' location (a retroverted uterus or bowel interference).

Group $2(n=77)$ showed an ineligibility percentage of $25 \%$ $(19 / 77)$ of which $0 \%(0 / 77)$ was due to bowel interference or a retroverted uterus (Table 1). Thus, our ineligibility rate due to the location of the uterine fibroid decreased from 18 to $0 \%$.

In total, we treated 67 women with 91 symptomatic uterine fibroids. The baseline characteristics of the patients are shown in Table 2 . The mean NPV\% immediately post-treatment was 


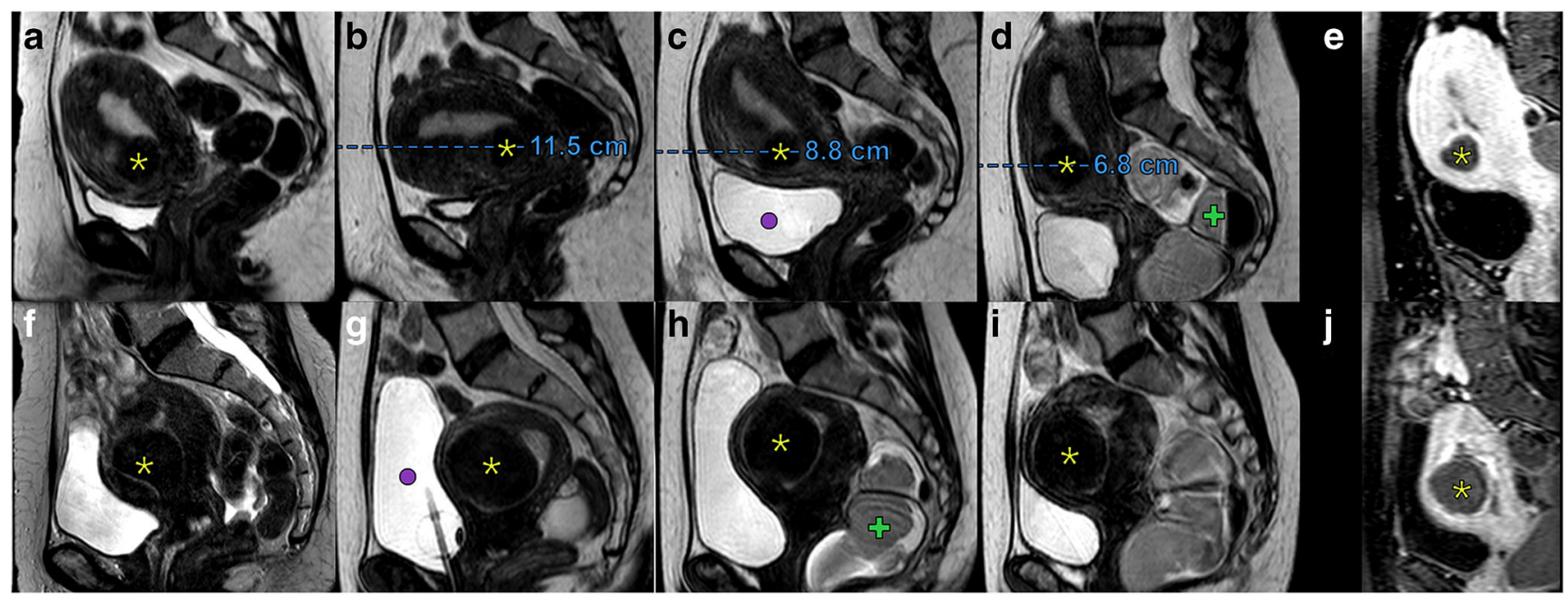

Fig. 2 The screening MR images are shown on the left (a and $\mathbf{f}$ ) of two different patients who underwent MR-HIFU therapy. On treatment day, manipulation techniques were applied to achieve optimal patient positioning. The pre-treatment T2w 3D images (b) of the first patient demonstrated an increased distance between the fibroid (yellow star) and the abdominal wall, contrary to our expectations based on the screening MRI (a). A combination of bladder filling (c; purple dot) and rectal filling with saline solution, ultrasound gel and psyllium fibers (d; green cross) moved the

$60.0 \pm 35.9 \%$. There were no complications or thermal injuries to the bowel or uterus reported. fibroid anteriorly. Bowel interference was expected in the second patient, based on the screening MR images of the targeted fibroid (f; yellow star). To displace the bowel loops, the BRB maneuver was used which included sequential applications of urinary bladder filling ( $\mathbf{g}$; purple dot), rectal filling (h; green cross), and bladder emptying (i). e, $\mathbf{j}$ The immediate post-HIFU contrast-enhanced T1w images. Specific treatment planning images are provided in the supplemental information

Group $1(n=20)$ received the following manipulation techniques (Table 3$)$ : urinary filling only $(n=1)$, rectal filling only

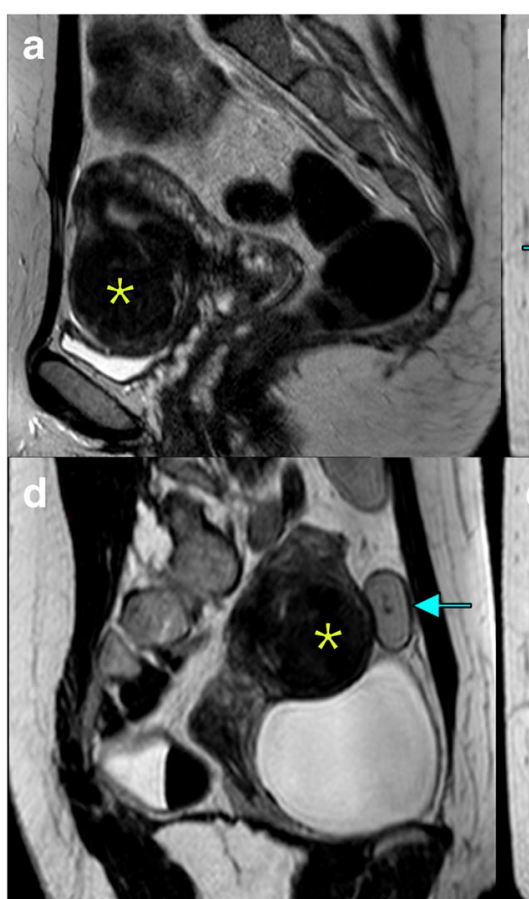

Fig. 3 A symptomatic uterine fibroid (yellow star) located in the anterior wall of the uterus of a patient. On the screening MR images (a), there was no interposition of bowel loops observed. However, on the MR-HIFU treatment day, manipulation techniques were necessary to prevent treatment failure due to the (unexpected) interposition of small bowel loops (turquoise arrows). First, the BRB maneuver (b and $\mathbf{c}$ ) was performed to displace the bowel loops. In some cases, the bladder filling will only cause an upwards movement of the uterus (c). Therefore, this patient

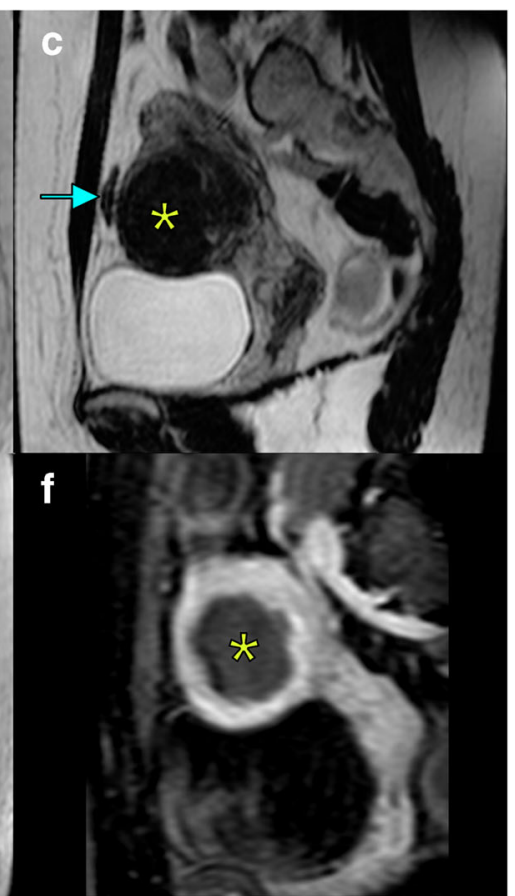

was placed into supine and Trendelenburg positions (d). Additionally, abdominal massage was performed on both sides of the lower abdomen with movements towards the upper abdomen $(\mathbf{d})$. After $5 \mathrm{~min}$, the patient was returned in prone position and the MR images no longer showed interposition of bowel loops (e). f Post-HIFU contrast-enhanced T1w image. Specific treatment planning images are provided in the supplemental information 


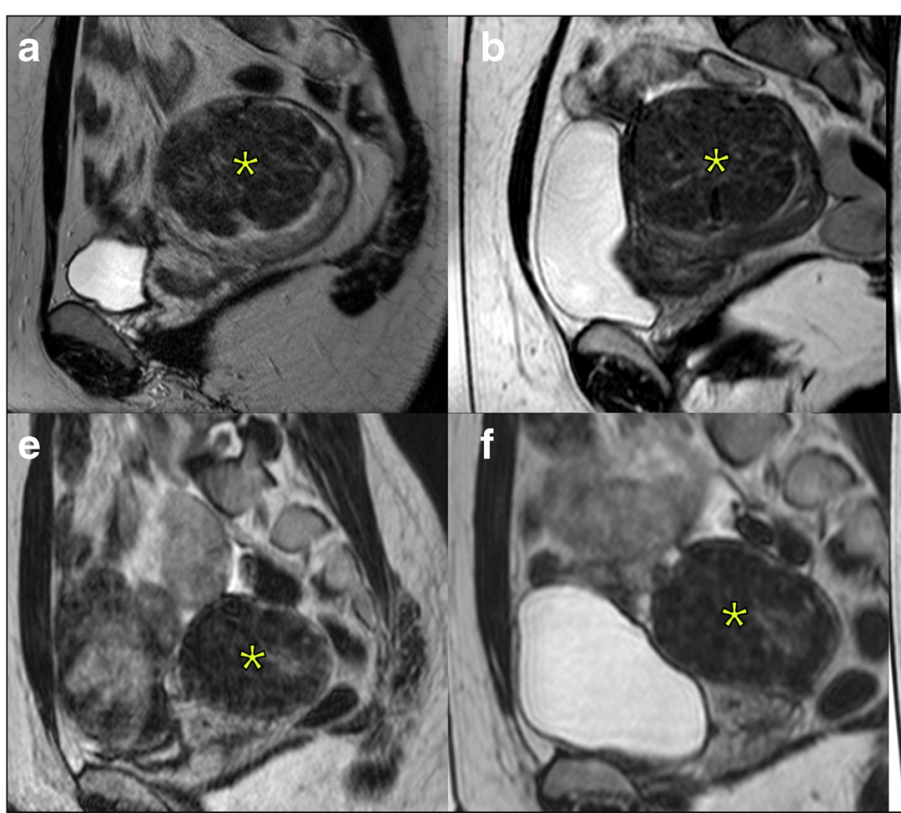

Fig. 4 The screening MR images (a and e) of two different cases are shown to illustrate the manual uterine manipulation (MUM) method. a The screening MR image of a uterine fibroid (yellow star) located in the retroverted uterus. To create an acoustic window for MR-HIFU treatment, anteversion of the uterus was required since bladder and rectal filling was not sufficient (b). The MUM method was used to reposition the uterus (c). In this case, rectal filling and a plastic speculum (blue arrowhead) were used for fixation of the uterine position. A speculum is necessary when the fibroid cannot be fixated with rectal filling only. e The screening MR

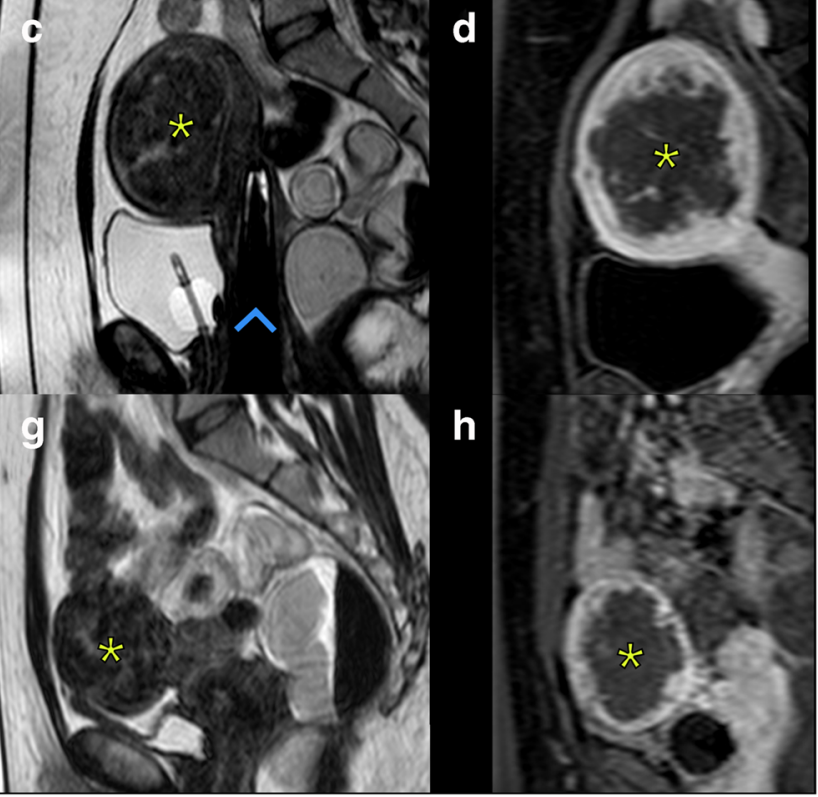

images of a retroverted uterus with a single pedunculated uterine fibroid (yellow star). Bladder filling was performed to prepare for manual repositioning (f); this prevents the interposition of bowel loops during the MUM method of the uterine fibroid. After anterior displacement of the uterine fibroid, rectal filling was used for fixation and the bladder was emptied (g), and the fibroid was accessible for MR-HIFU therapy. $\mathbf{d}, \mathbf{h}$ The immediate post-HIFU contrast-enhanced T1w images. Specific treatment planning images are provided in the supplemental information

Table 1 Reasons for ineligibility of patients for MR-HIFU based on screening MR images: a comparison between two manipulation treatment protocols

\begin{tabular}{lcl}
\hline Reason for ineligibility & $\begin{array}{l}\text { Group A: BRB } \\
\text { maneuver } \\
\text { protocol } \\
(n=88 / 165 \\
\text { screened })\end{array}$ & $\begin{array}{l}\text { Group B: 3-step } \\
\text { modified manipulation } \\
\text { protocol }(n=77 / 165 \\
\text { screened })\end{array}$ \\
\hline Subcutaneous fat layer $>4 \mathrm{~cm}$ & $3(3.4)$ & $4(5.2)$ \\
Fibroid size & $3(3.4)$ & $2(2.6)$ \\
$>10$ fibroids & $8(2.4)$ & $1(1.3)$ \\
Adenomyosis (concomitant) & $4(4.5)$ & $7(9.1)$ \\
No enhancement on CE-T1WI & $1(1.1)$ & $2(2.6)$ \\
Large abdominal scar & $1(1.1)$ & $0(0.0)$ \\
Excessively high signal & $11(12.5)$ & $1(1.3)$ \\
$\quad$ intensity on T2WI & $6(6.8)$ & $0(0.0)$ \\
Uterus RVF & $10(11.4)$ & $0(0.0)$ \\
Fibroid's location/interposition & $0(0.0)$ & $1(1.3)$ \\
Claustrophobia & $0(0.0)$ & $1(1.3)$ \\
Possible malignancy & $47 / 88(53.4)$ & $19 / 77(24.7)$ \\
Total ineligible & & \\
\hline
\end{tabular}

Data presented as $n(\%)$

$B R B$, bladder filling, rectal filling, bladder emptying; $C E-T 1 W I$, contrastenhanced T1-weighted imaging; T2WI, T2-weighted imaging; $R V F$, retroverted position of the uterus 
Table 2 Baseline patient characteristics

Characteristic

\begin{tabular}{|c|c|c|}
\hline Age (years) & & $44.0 \pm 7.0$ \\
\hline BMI $\left(\mathrm{kg} / \mathrm{m}^{2}\right)$ & & $24.98 \pm 3.56$ \\
\hline Subcutaneous fat layer $(\mathrm{cm})$ & & $2.16 \pm 1.11$ \\
\hline \multirow[t]{2}{*}{ History of pregnancy } & Yes & $41(61.2)$ \\
\hline & No & $26(38.8)$ \\
\hline \multirow[t]{2}{*}{ History of prior medical treatment } & Yes & $44(65.7)$ \\
\hline & No & $23(34.3)$ \\
\hline Abdominal scar present & & $12(18.2)$ \\
\hline Baseline tSSS & & $49.9 \pm 16.3$ \\
\hline Baseline tHRQL & & $57.5 \pm 19.9$ \\
\hline \multirow[t]{2}{*}{ Uterine position } & Anteverted & $55(82.1)$ \\
\hline & Retroverted & $12(17.9)$ \\
\hline \multirow[t]{6}{*}{ Number of fibroids } & 1 & $27(40.3)$ \\
\hline & 2 & $14(20.9)$ \\
\hline & 3 & $13(19.4)$ \\
\hline & 4 & $4(6.0)$ \\
\hline & 5 & $4(6.0)$ \\
\hline & $>5$ & $5(7.5)$ \\
\hline \multirow[t]{5}{*}{ Number of fibroids treated } & 1 & $56(83.5)$ \\
\hline & 2 & $4(6.0)$ \\
\hline & 3 & $5(7.5)$ \\
\hline & 4 & $1(1.5)$ \\
\hline & 5 & $1(1.5)$ \\
\hline \multirow[t]{4}{*}{ Fibroid location (FIGO) } & Submucosal & $24(26.4)$ \\
\hline & Intramural & $23(25.3)$ \\
\hline & Submucosal & $29(31.9)$ \\
\hline & Hybrid & $15(16.5)$ \\
\hline \multirow[t]{3}{*}{ Funaki classification } & 1 & $8(8.8)$ \\
\hline & 2 & $74(81.3)$ \\
\hline & 3 & $9(9.9)$ \\
\hline Fibroid volume $\left(\mathrm{cm}^{3}\right)$ & & $133.10 \pm 207.06$ \\
\hline Maximum diameter $(\mathrm{cm})$ & & $5.78 \pm 3.29$ \\
\hline
\end{tabular}

Data presented as mean \pm standard deviation or $n(\%)$

$B M I$, body mass index; $t S S S$, transformed Symptom Severity Score; tHRQL, transformed Health-Related Quality of Life; FIGO, Fédération Internationale de Gynécologie et d'Obstétrique

loops were seen in the sonication pathway cranially from the uterine fibroid.

By implementing the 3-step modified manipulation protocol, our treatment failure rate due to the interposition of bowel loops decreased from $20 \%(4 / 20)$ to $2 \%(1 / 47)$. Only $2 \%$ (1/47) of the MR-HIFU treatments failed because the 3-step modified manipulation protocol was not sufficient to provide an unobstructed acoustic window on the fibroid (Table 4). In this specific case, the fibroid was located in the recto-sigmoid region while the uterus was already anteverted. Eventually, this patient was treated successfully because an endo-rectal
Table 3 Comparison of the manipulation techniques for MR-HIFU treatments using the two different manipulation protocols

\begin{tabular}{lcc}
\hline $\begin{array}{l}\text { Manipulation } \\
\text { techniques }\end{array}$ & $\begin{array}{l}\text { Group 1: BRB } \\
\text { maneuver } \\
(n=20 / 67)\end{array}$ & $\begin{array}{l}\text { Group 2: 3-step } \\
\text { modified manipulation } \\
\text { protocol }(n=47 / 67)\end{array}$ \\
\hline None & $6(30.0)$ & $17(36.2)$ \\
Manipulation with: & $14(70.0)$ & $29(61.7)$ \\
- Urinary filling & $1(5.0)$ & $0(0.0)$ \\
- Rectal filling & $1(5.0)$ & $4(8.5)$ \\
- BRB & $12(60.0)$ & $12(25.5)$ \\
- Trendelenburg + & $0(0.0)$ & $4(8.5)$ \\
bowel massage & $0(0.0)$ & $8(17.0)$ \\
- MUM & $0(0.0)$ & $1(2.1)$ \\
- Extra gel pad & $0(0.0)$ & $1(2.1)$ \\
- Endo-rectal coil & & \\
\hline
\end{tabular}

Data presented as $n(\%)$

$B R B$, bladder filling, rectal filling, bladder emptying; $M U M$, manual uterine manipulation

coil filled with saline solution was inserted which caused anterior displacement of the uterine fibroid (Fig. 5).

\section{Discussion}

The aim of this study was to evaluate the effect of a 3-step modified manipulation protocol developed by us to improve the eligibility percentage and treatment failure rate of MRIHIFU for uterine fibroids.

After implementing our 3-step modified manipulation protocol, the location of the uterine fibroid on screening MR images was no longer a reason for ineligibility which drastically improved our eligibility percentage. An eligibility percentage of $75 \%$ is one of the highest reported to date $[1,2]$. Although Zaher et al reported that $74 \%$ of the patients in their study was suitable for MR-HIFU, patients in their study with large or multiple fibroids were not excluded and $38 \%$ required two MR-HIFU sessions [7]. A more recently published study also described the use of bowel interference mitigation strategies and reported an eligibility percentage of $72 \%[7,9]$. With more liberal inclusion criteria, MR-HIFU treatment can be offered to the majority of the referred patients with symptomatic uterine fibroids.

Comparison of treatment failures due to unsuccessful manipulation between the two protocols showed a reduction of $18 \%$, which is an important improvement from a clinical perspective. This result may be biased by the fact that a learning curve (negatively) influenced our treatments in group 1 [13]. However, it is also plausible that the patients in group 2 were more difficult manipulation cases because a difficult location of the fibroid was no reason for exclusion. The learning curve also coincided with the decision that not all Funaki type 3 fibroids 
Table 4 Reasons for treatment failures compared between the two manipulation protocols for MR-HIFU therapy

\begin{tabular}{lll}
\hline Reason for failure & $\begin{array}{l}\text { Group 1: BRB } \\
\text { maneuver }(n=20 / 67)\end{array}$ & $\begin{array}{l}\text { Group 2: 3-step } \\
\text { modified manipulation } \\
\text { protocol }(n=47 / 67)\end{array}$ \\
\hline Interposition & $4(20.0)$ & $1(2.1)$ \\
Scar patch & $2(10.0)$ & $0(0.0)$ \\
Motion artifacts & $1(5.0)$ & $0(0.0)$ \\
Inadequate heating & $1(5.0)$ & $3(6.4)$ \\
Patient discomfort & $0(0.0)$ & $1(2.1)$ \\
Device malfunction & $0(0.0)$ & $1(2.1)$ \\
Total & $8 / 20(40.0)$ & $6 / 47(12.8)$ \\
\hline
\end{tabular}

Data presented as $n(\%)$

$B R B$, bladder filling, rectal filling, bladder emptying

were excluded in group 2. This decision, as expected, led to more treatment failures due to inadequate heating. Dropping this exclusion criterion negatively influenced the treatment failure rate in group 2. Despite all the abovementioned more liberal inclusion criteria, we still managed to reduce our overall treatment failure rate and we eliminated the problem of bowel interference (in a relatively high-volume experienced MR-HIFU center).

To share our experience with the different manipulation techniques applied in this study, we will discuss the details of each approach further below.

To prevent the displaced bowel loops from moving downwards, maintenance of urinary bladder filling was sometimes necessary. This is not desirable because a clamped Foley catheter results in an upwards movement of the uterine fibroid due to inflow of urine. This may lead to misregistration between the real location of the fibroid and the treatment planning images; this can be monitored by placing markers. To solve this issue, renewed $\mathrm{T} 2 \mathrm{w}$ planning images can be obtained in between sonications, although this is lengthening the procedure. However, the already performed sonications will remain at their original (incorrect) position due to misregistration. This could lead to partially ablated fibroids, but development of MR registration could overcome this problem, and therefore enhance treatment efficacy.

In our experience, rectal filling with ultrasound gel only was suboptimal because the gel slowly migrates proximally which led to displacement of the uterine fibroid during the therapy. Park et al reported that the rectal distention was usually maintained for almost an hour [10], but the duration of the MR-HIFU therapy is generally a couple of hours. Therefore, psyllium fibers were added to the fluid used for rectal filling. Psyllium fibers have been widely used for radiological examinations of the bowel as a water-soluble contrast agent [14]. The adjusted rectal filling applied firm pressure during the total length of the MR-HIFU procedure. Moreover, the uterine fibroid was displaced more anteriorly when psyllium fibers were used. Compared with Park et al, the total amount of rectal filling was much larger, which our patients tolerated easily without complaining of a sense of defecation or constipation afterwards [10]. Post-treatment, patients were instructed to drink lots of water to prevent constipation from the psyllium fibers.

To the best of our knowledge, the MUM method has not been described elsewhere. Pulanic et al did report that vaginal pessary placement may help in uterine repositioning to enable safe ablation [15]. Jeong et al demonstrated the feasibility of an uterine elevator device to change the position of the uterus, but this requires a MRI-compatible uterine manipulator device which must be sutured in the cervix pre-treatment [16]. Compared with the uterine elevator, MUM has the advantage of being non-invasive and cost free. Another advantage of MUM was the provided real-time haptic feedback during manual repositioning. With other manipulation techniques, new MR images have to be obtained to visualize the effect. Logically, MUM was the fastest manipulation method. PostHIFU, patients did not remember the manipulation procedure

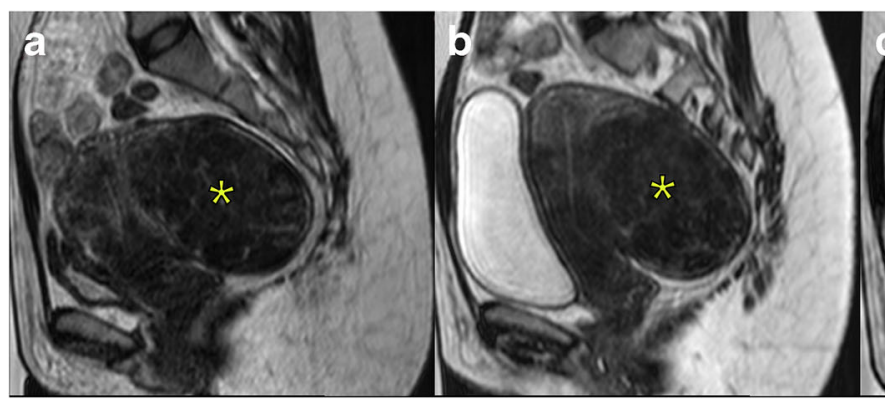

Fig. 5 Pre-treatment MR images showed a uterine fibroid located on the posterior wall of an anteverted uterus (a). The image on the left shows the position of the fibroid without manipulation (yellow star). The BRB and MUM techniques both failed to move the uterine fibroid anteriorly (b). Therefore, a rectal balloon device (prostate endo-rectal coil; orange square) was inserted (c). The endo-rectal coil was filled with water and

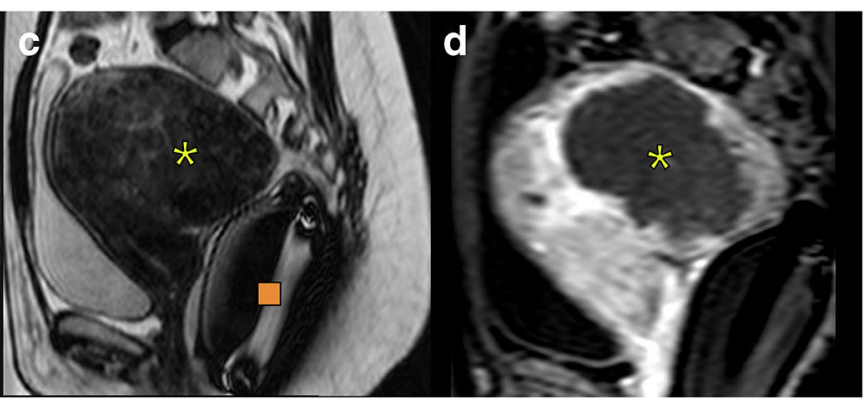

surrounded by the ultrasound gel. The inflatable endo-rectal device caused anterior displacement of the uterine fibroid which is now accessible for MR-HIFU treatment. d The post-HIFU contrast-enhanced T1w image. Specific treatment planning images are provided in the supplemental information 
itself due to the moderate-deep sedation with propofol. Importantly, the renewed position of the uterine fibroid was maintained during the complete MR-HIFU procedure. This is probably due to a combination of the effect of gravity while in the prone position and the fixation with rectal filling and/or a plastic speculum.

Miscellaneous bowel interference mitigation strategies have been described such as the use of a degassed water balloon and a wedged gel pad [17-19]. In this work, we share our experience with the Trendelenburg maneuver and bowel massage. Both were the most time consuming of all different manipulation techniques since the patient must be turned into supine position; but in some cases, it may be the only option to prevent treatment failure. Therefore, we only performed this step when the previous options failed.

In one patient, the current 3-step modified manipulation protocol failed to create an appropriate acoustic window. Nonetheless, this patient was treated successfully by using an endo-rectal coil for anterior displacement of the uterine fibroid. The use of an endo-rectal coil is not a standard procedure at our center since it is expensive and sensitive to artifacts, but it was helpful in treating this large fibroid located in the recto-sigmoid region. The use of another endo-rectal device without a coil, but with a cooling system, has been previously described for MR-HIFU prostate treatment with the TULSA-PRO system [20]. However, this device is not inflatable and may therefore not provide enough pressure to move the fibroid anteriorly. Development of an endo-rectal balloon device with a cooling system would be the ideal tool for manipulation since it could also potentially protect the bowel from thermal damage. Thermal complications such as bowel perforation are very rare but have been reported in literature after HIFU of uterine fibroids [21-23]. Development of an endo-rectal balloon device (without coil or cooling) would be a more cost-effective solution.

In our opinion, adequate patient preparation is key for a successful MR-HIFU treatment. Technological tools have been developed to overcome the issue of bowel interference such as beam-shaping and angulation, but these tools further limit the focal depth and power of the system which often lead to partially unreachable fibroids or lengthening of the procedure [8]. Despite the additional time necessary before sonicating, optimal patient positioning reduced the risk for treatment failure.

A limitation of this work was the relatively small sample size and the single-center design. Dissemination and implementation of the 3-step modified manipulation protocol to other institutions in clinical practice will be challenging. Moreover, a learning curve effect complicated the evaluation of our results which may have distorted the comparison between manipulation protocols. Therefore, a larger multi-center cohort study should be conducted in the future to follow-up on implementation and the results of the 3-step modified manipulation protocol.

\section{Conclusions}

Implementation of a newly developed 3-step modified manipulation protocol increased the eligibility percentage and reduced the treatment failure rate for treatment of uterine fibroids with MR-HIFU.

Funding information The authors state that this work has not received any funding.

\section{Compliance with ethical standards}

Guarantor The scientific guarantor of this publication is M.F. Boomsma, MD, PhD.

Conflict of interest The authors of this manuscript declare no relationships with any companies whose products or services may be related to the subject matter of the article.

Statistics and biometry No complex statistical methods were necessary for this paper.

Informed consent Written informed consent was obtained from all subjects (patients) in this study.

Ethical approval Institutional Review Board approval was obtained.

Methodology

- Prospective

- Experimental

- Performed at one institution

\section{References}

1. Pron G (2015) Magnetic resonance-guided high-intensity focused ultrasound (MRgHIFU) treatment of symptomatic uterine fibroids: an evidence-based analysis. Ont Health Technol Assess Ser 15:186

2. Verpalen IM, Anneveldt KJ, Nijholt IM et al (2019) Magnetic resonance-high intensity focused ultrasound (MR-HIFU) therapy of symptomatic uterine fibroids with unrestrictive treatment protocols: a systematic review and meta-analysis. Eur J Radiol 120: 108700. https://doi.org/10.1016/j.ejrad.2019.108700

3. Duc NM, Keserci B (2018) Review of influential clinical factors in reducing the risk of unsuccessful MRI-guided HIFU treatment outcome of uterine fibroids. Diagn Interv Radiol 24:283-291. https:// doi.org/10.5152/dir.2018.18111

4. Fröling V, Kröncke TJ, Schreiter NF et al (2014) Technical eligibility for treatment of magnetic resonance-guided focused ultrasound surgery. Cardiovasc Intervent Radiol 37:445-450. https:// doi.org/10.1007/s00270-013-0678-Z

5. Behera MA, Leong M, Johnson L, Brown H (2010) Eligibility and accessibility of magnetic resonance-guided focused ultrasound (MRgFUS) for the treatment of uterine leiomyomas. Fertil Steril 94:1864-1868. https://doi.org/10.1016/j.fertnstert.2009.09.063 
6. Arleo EK, Khilnani NM, Ng A, Min RJ (2007) Features influencing patient selection for fibroid treatment with magnetic resonanceguided focused ultrasound. J Vasc Interv Radiol 18:681-685. https://doi.org/10.1016/j.jvir.2007.02.015

7. Zaher S, Gedroyc WM, Regan L (2009) Patient suitability for magnetic resonance guided focused ultrasound surgery of uterine fibroids. Eur J Obstet Gynecol Reprod Biol 143:98-102. https:// doi.org/10.1016/j.ejogrb.2008.12.011

8. Kim YS, Bae DS, Park MJ et al (2014) Techniques to expand patient selection for MRI-guided high-intensity focused ultrasound ablation of uterine fibroids. AJR Am J Roentgenol 202:443-451. https://doi.org/10.2214/AJR.13.10753

9. Kim YS, Lim HK, Rhim H (2016) Magnetic resonance imagingguided high-intensity focused ultrasound ablation of uterine fibroids: effect of bowel interposition on procedure feasibility and a unique bowel displacement technique. PLoS One 11:e0155670. https://doi.org/10.1371/journal.pone.0155670

10. Park MJ, Kim YS, Rhim H, Lim HK (2013) Technique to displace bowel loops in MRI-guided high-intensity focused ultrasound ablation of fibroids in the anteverted or anteflexed uterus. AJR Am J Roentgenol 201:W761-W764. https://doi.org/10.2214/AJR.12. 10081

11. Spies JB, Coyne K, Guaou Guaou N, Boyle D, Skyrnarz-Murphy K, Gonzalves SM (2002) The UFS-QOL, a new disease-specific symptom and health-related quality of life questionnaire for leiomyomata. Obstet Gynecol 99:290-300. https://doi.org/10. 1016/s0029-7844(01)01702-1

12. Funaki K, Fukunishi H, Funaki T, Sawada K, Kaji Y, Maruo T (2007) Magnetic resonance-guided focused ultrasound surgery for uterine fibroids: relationship between the therapeutic effects and signal intensity of preexisting T2-weighted magnetic resonance images. Am J Obstet Gynecol 196:184.e1-184.e6. https://doi.org/10. 1016/j.ajog.2006.08.030

13. Okada A, Morita Y, Fukunishi H, Takeichi K, Murakami T (2009) Non-invasive magnetic resonance-guided focused ultrasound treatment of uterine fibroids in a large Japanese population: impact of the learning curve on patient outcome. Ultrasound Obstet Gynecol 34:579-583. https://doi.org/10.1002/uog.7454

14. Saini S, Colak E, Anthwal S, Vlachou PA, Raikhlin A, Kirpalani A (2014) Comparison of 3\% sorbitol vs psyllium fibre as oral contrast agents in MR enterography. Br J Radiol 87:20140100. https://doi. org $/ 10.1259 /$ bjr.20140100

15. Klepac-Pulanic T, Venkatesan AM, Segars J, Sokka S, Wood BJ, Stratton P (2016) Vaginal pessary for uterine repositioning during high-intensity focused ultrasound ablation of uterine leiomyomas. Gynecol Obstet Invest 81:285-288. https://doi.org/10.1159/ 000441782

16. Jeong JH, Hong KP, Kim YR, Ha JE, Lee KS (2017) Usefulness of modified BRB technique in treatment to ablate uterine fibroids with magnetic resonance image-guided high-intensity focused ultrasound. Obstet Gynecol Sci 60:92-99. https://doi.org/10.5468/ogs. 2017.60.1.92

17. Zhang L, Chen W, Liu Y et al (2010) Feasibility of magnetic resonance imaging-guided high intensity focused ultrasound therapy for ablating uterine fibroids in patients with bowel lies anterior to uterus. Eur J Radiol 73:396-403. https://doi.org/10.1016/j.ejrad.2008. 11.002

18. Sainio T, Komar G, Saunavaara J et al (2018) Wedged gel pad for bowel manipulation during MR-guided high-intensity focused ultrasound therapy to treat uterine fibroids: a case report. J Ther Ultrasound 6:10. https://doi.org/10.1186/s40349-018-0116-4

19. Hesley GK, Felmlee JP, Gebhart JB et al (2006) Noninvasive treatment of uterine fibroids: early mayo clinic experience with magnetic resonance imaging-guided focused ultrasound. Mayo Clin Proc 81:936-942. https://doi.org/10.4065/81.7.936

20. Bonekamp D, Wolf MB, Roethke MC et al (2019) Twelve-month prostate volume reduction after MRI-guided transurethral ultrasound ablation of the prostate. Eur Radiol 29:299-308. https://doi. org/10.1007/s00330-018-5584-y

21. Ko JKY, Seto MTY, Cheung VYT (2018) Thermal bowel injury after ultrasound-guided high-intensity focused ultrasound treatment of uterine adenomyosis. Ultrasound Obstet Gynecol 52:282-283. https://doi.org/10.1002/uog.18965

22. Hwang DW, Song HS, Kim HS, Chun KC, Koh JW, Kim YA (2017) Delayed intestinal perforation and vertebral osteomyelitis after high-intensity focused ultrasound treatment for uterine leiomyoma. Obstet Gynecol Sci 60:490-493. https://doi.org/10. 5468/ogs.2017.60.5.490

23. Chen J, Chen W, Peng S et al (2015) Safety of ultrasound-guided ultrasound ablation for uterine fibroids and adenomyosis: a review of 9988 cases. Ultrason Sonochem 27:671-676. https://doi.org/10. 1016/j.ultsonch.2015.05.031

Publisher's note Springer Nature remains neutral with regard to jurisdictional claims in published maps and institutional affiliations. 